\title{
Analysis of Families at Risk for Insulin-Dependent Diabetes Mellitus Reveals that HLA Antigens Influence Progression to Clinical Disease
}

\author{
Margo C. Honeyman*, Leonard C. Harrison*, \\ Bruce Drummond ${ }^{\dagger}$, Peter G. Colman ${ }^{\dagger}$, and Brian D. Tait ${ }^{\dagger}$ \\ *Burnet Clinical Research Unit, Walter and Eliza Hall Institute of \\ Medical Research \\ ${ }^{\dagger}$ Royal Melbourne Hospital, Parkville, Victoria, Australia
}

\begin{abstract}
Background: Individuals at risk for insulin-dependent diabetes mellitus (IDDM), with an affected first-degree relative, can be identified by the presence of islet cell antibodies (ICA). ICA-positive relatives progress at variable rates to IDDM and identification of those at highest risk is a prerequisite for possible preventative treatment. Those who develop IDDM may exhibit less genetic heterogeneity than their ICA-positive or ICA-negative relatives. Specific human leucocyte antigen (HLA) genes predispose to IDDM but could also influence the rate of progression of preclinical disease. Therefore, by comparing HLA antigen frequencies between first-degree relatives, we sought to identify HLA genes that influence progression to IDDM.

Materials and Methods: HLA antigen frequencies were compared in 68 IDDM, 53 ICA-positive, and 96 ICA-negative first-degree relatives from 40 Caucasoid families. Predictions were tested in a panel of 270 unrelated IDDM subjects. HLA typing was performed serologically (HLA class I and II) and by sequence-specific oligotyping (11th International Histocompatibility Workshop protocol) (HLA class II). ICA tests were measured by an internationally standardized indirect immunofluorescence assay.

Results: In general, known susceptibility class II HLA

class II HLA antigens decreased in frequency, from ICAnegative to ICA-positive to IDDM relatives. Thus, DR4 and DQ8 increased whereas DR2 and DQ6 decreased; DR3 and DQ2 increased from ICA-negative to ICA-positive relatives, but not further in IDDM relatives. The high-risk DR3, 4 phenotype increased across the three groups; DR4,X was unchanged, and DR3,X and DRX, X both decreased. The HLA class I antigen, A24, occurred more frequently in ICA-positive relatives who developed IDDM and, in 270 unrelated IDDM subjects, was associated with an earlier age at diagnosis of IDDM in those with the lower risk class II phenotypes DR4,4 and DR3,X. Conclusions: HLA-DR3 and DQ2 predispose to islet autoimmunity, but not development of clinical IDDM in the absence and DR4 and DQ8. DR4 and DQ8 predispose to the development of clinical IDDM in ICA-positive relatives, in combination with DR3-DQ2 and other haplotypes but not when homozygous. HLA-A24 is significantly associated with rapid progression to IDDM in ICA-positive relatives and with an earlier age at clinical diagnosis. Analysis of IDDM families reveals that HLA genes not only predispose to islet autoimmunity but influence progression to clinical disease. The findings have implications for identifying high-risk relatives as candidates for possible preventative therapy.
\end{abstract} antigens increased in frequency and known protective

\section{INTRODUCTION}

Insulin-dependent diabetes mellitus (IDDM) results from $\mathrm{T}$ cell-mediated destruction of pancreatic islet beta cells (1). Individuals at risk for

Address correspondence and reprint requests to: Margo $\mathrm{C}$. Honeyman, Burnet Clinical Research Unit, Walter and Eliza Hall Institute of Medical Research, Post Office, Royal Melbourne Hospital, Parkville, Victoria, 3050, Australia.
IDDM can be identified by the presence of circulating islet cell antibodies (ICA), but only a proportion of them progress to clinical disease within a decade of follow-up (2). Identifying factors that determine the rate of progression through the preclinical stage is important for accurate prediction and prevention of clinical disease. Evidence from the nonobese diabetic (NOD) mouse suggests that progression to diabe- 
tes mellitus is a staged process regulated by different genes (3). In humans, the major known component of genetic risk for IDDM is contributed by the HLA genes of the major histocompatibility complex (MHC) (4). In Caucasoids, the highest risk is associated with haplotypes bearing the HLA class II alleles DR3-DQ2 and DR4-DQ8, and the lowest with DR2-DQ6 (4). Independently of these class II haplotypes, no HLA class I allele has been shown to confer risk. An intriguing association of specific HLA antigens with age at clinical diagnosis $(5,6)$ suggests that HLA genes may influence the rate of beta cell destruction. In first-degree relatives in families with IDDM, the risk for disease increases step-wise, if they share one then two HLA haplotypes with the proband $(7,8)$.

Against this background, individuals who have developed IDDM might be expected to display less HLA genetic heterogeneity than their healthy, ICA-positive "at risk" and particularly their normal, ICA-negative first-degree relatives. Comparison of HLA antigen frequencies between IDDM relatives and ICA-positive and -negative relatives could therefore delineate HLA genes that predispose to the initiation of islet autoimmunity from those that influence the progression to clinical IDDM. To explore this possibility, we analyzed the distribution of HLA antigen frequencies in 217 members of 40 IDDM families and tested predictions in a panel of 270 unrelated IDDM subjects.

\section{SUBJECTS AND METHODS}

First-degree relatives in 40 IDDM families from the Melbourne Prediabetes mellitus Study were grouped as follows: 96 healthy, ICA-negative (53 parents, 43 children); 53 healthy, ICA-positive (22 parents, 31 children); and 68 diabetic (12 parents, 56 children).

HLA-A, B, DR, and DQ typing was performed by a standard serologically based microlymphocytotoxicity assay. T (class I) and B (class II) lymphocytes were separated by a nylon wool column method or by antibody-coated magnetic beads. Sequence specific oligotyping according to the 11 th International Histocompatibility protocol (9) was used to confirm diabetes mellitus-associated DQ alleles in families. Four hundred seventeen healthy Australian blood donors served as unrelated controls. Predictions from the family data were tested on a panel of
270 IDDM subjects attending clinics at the Royal Melbourne Hospital.

Sera from all 217 relatives were tested for ICA by an internationally standardized indirect immunofluorescence assay using sections of human type 0 pancreata. Subjects with ICA $\geq 20$ JDF units were selected because those with ICA $<20$ alone do not have a significantly increased risk for IDDM within a decade.

Statistical analysis by the $2 \times 2 \chi^{2}$ test with Yates' correction was used to test the null hypothesis that no difference in HLA antigen frequencies existed between relatives comprising the different groups. All relatives, including HLA identical siblings, were included in the data set because the null hypothesis predicts that all have an equal opportunity to be either ICA-negative, ICA-positive, or diabetic. The caveat is that some of the ICA-negative subjects may still be too young to have entered the other groups. Their presence in the ICA-negative group would, however, only bias HLA frequencies towards similarity, rather than differences, between groups. $p$ values were not corrected if the antigen in question had previously been associated with IDDM. Comparisons of ages at diagnosis within the unrelated IDDM subjects were made using the Wilcoxon rank sum test for nonparametric data. Comparisons of DR phenotype frequencies between family and unrelated IDDM subjects were made using a $2 \times 6 \chi^{2}$ test.

\section{RESULTS}

\section{HLA Class II Alleles}

The frequencies of HLA-DR3 and DR4 were higher in each group of relatives (DR3, $p<$ 0.0005 ; DR4, $p<0.005-<0.0005)$ than in unrelated controls, and lower in ICA-negative than in IDDM relatives (DR3 $47 \%$ versus $65 \%, p<0.05$; DR4 $50 \%$ versus $76 \%, p<0.005$ ) with ICApositive relatives being intermediate (DR3 60\%, DR4 66\%) (Table 1). The frequency of DR2 did not differ between unrelated controls and ICAnegative relatives $(27 \%$ versus $23 \%)$, but was lower in the IDDM relatives than in unrelated controls and ICA-negative relatives $(1 \%, p<$ $0.0005,<0.005)$ with ICA-positive relatives being intermediate $(9 \%)$.

Because there is synergy between DR3 and DR4 in predisposing to IDDM, HLA-DR phenotypes that included these risk alleles were also compared between family groups (Table 2 ). The 
TABLE 1. Frequencies of HLA antigens which vary within IDDM families

\begin{tabular}{|c|c|c|c|c|c|c|c|c|}
\hline \multirow[b]{3}{*}{ Antigens } & \multirow{2}{*}{\multicolumn{2}{|c|}{$\begin{array}{l}\text { Unrelated } \\
\text { Controls }\end{array}$}} & \multicolumn{6}{|c|}{ IDDM Family Group } \\
\hline & & & \multicolumn{2}{|c|}{ ICA-Negative } & \multicolumn{2}{|c|}{ ICA-Positive } & \multicolumn{2}{|c|}{ IDDM } \\
\hline & $n$ & $\%$ & $n$ & $\%$ & $n$ & $\%$ & $n$ & $\%$ \\
\hline DR4 & 138 & 33 & $48^{a}$ & 50 & $35^{b}$ & 66 & $52^{b, c}$ & 76 \\
\hline DR3 & 113 & 27 & $45^{b}$ & 47 & $32^{b}$ & 60 & $44^{b, d}$ & 65 \\
\hline DR2 & 113 & 27 & 22 & 23 & $5^{e}$ & 9 & $1^{b, c}$ & 1 \\
\hline DQ8 & 100 & 24 & $37^{f}$ & 38 & $24^{a}$ & 45 & $40^{b, g}$ & 59 \\
\hline DQ7 & 150 & 36 & $20^{f}$ & 21 & $10^{h}$ & 19 & $14^{f}$ & 20 \\
\hline DQ2 & 191 & 46 & 49 & 51 & $36^{a}$ & 68 & $46^{a, d}$ & 68 \\
\hline DQ6 & 42 & 10 & 11 & 11 & 3 & 6 & $1^{d, f}$ & 1 \\
\hline B8 & 104 & 25 & $36^{h}$ & 38 & $25^{a}$ & 47 & $30^{a}$ & 44 \\
\hline $\mathrm{Al}$ & 138 & 33 & 37 & 39 & $29^{a}$ & 55 & $31^{f}$ & 46 \\
\hline $\mathrm{A} 2$ & 208 & 50 & 46 & 48 & 28 & 53 & $23^{f}$ & 34 \\
\hline A24 & 67 & 16 & 23 & 24 & $5^{d}$ & 9 & $20^{f, i}$ & 29 \\
\hline
\end{tabular}

$p$ values compared with unrelated controls: ${ }^{a}<0.005,{ }^{b}<0.0005,{ }^{e}<0.01,{ }^{f}<0.05,{ }^{h}<0.025$.

$p$ values compared with ICA-negative relatives: ${ }^{c}<0.005,{ }^{d}<0.05,{ }^{g}<0.025$.

$p$ values compared with ICA-positive relatives: ${ }^{i}<0.025$.

frequency of DR 3,4 was lower in ICA-negative than in IDDM relatives ( $15 \%$ versus $44 \%, p<$ $0.0005)$ with antibody positive relatives being intermediate $(30 \%)$. The combined frequency of phenotypes homozygous for the known risk antigens DR3 and DR4 was lower in ICA-negative relatives than in IDDM relatives $(4+5=9 \%$ versus $10+12=22 \%, p<0.05$ ), being inter- mediate in ICA-positive healthy relatives $(6+7$ $=13 \%)$. The frequencies of phenotypes comprising a single risk antigen and nonrisk antigen (DR3,X and DR4,X) differed according to the risk antigen. Thus, the frequency of DR3, $X$ was higher in ICA-negative than in IDDM relatives ( $28 \%$ versus $10 \%, p<0.025)$, but was similar in ICA-positive and ICA-negative relatives $(28 \%$

TABLE 2. Frequency of DR Phenotypes

\begin{tabular}{|c|c|c|c|c|c|c|c|c|}
\hline \multirow[b]{3}{*}{ Phenotype } & \multicolumn{6}{|c|}{ IDDM Family Group } & \multirow{2}{*}{\multicolumn{2}{|c|}{$\begin{array}{c}\text { Unrelated } \\
\text { IDDM } \\
\text { Subjects } \\
(n=270)\end{array}$}} \\
\hline & \multicolumn{2}{|c|}{$\begin{array}{c}\text { ICA-Negative } \\
(n=96)\end{array}$} & \multicolumn{2}{|c|}{$\begin{array}{c}\text { ICA-Positive } \\
(n=53)\end{array}$} & \multicolumn{2}{|c|}{$\begin{array}{l}\text { IDDM } \\
(n=68)\end{array}$} & & \\
\hline & $n$ & $\%$ & $n$ & $\%$ & $n$ & $\%$ & $n$ & $\%$ \\
\hline DR 3,4 & 14 & 15 & 16 & 30 & 30 & 44 & 107 & 40 \\
\hline DR 3,3 & 4 & 4 & 3 & 6 & 7 & 10 & 26 & 10 \\
\hline DR4, 4 & 5 & 5 & 4 & 7 & 8 & 12 & 24 & 9 \\
\hline $\mathrm{DR} 4, \mathrm{X}^{a}$ & 30 & 31 & 15 & 28 & 14 & 21 & 64 & 24 \\
\hline DR3,X & 27 & 28 & 13 & 25 & 7 & 10 & 22 & 8 \\
\hline DRX,X & 16 & 17 & 2 & 4 & 2 & 3 & 27 & 10 \\
\hline
\end{tabular}

${ }^{a} \mathrm{X}=$ non-DR3, non-DR4. 
versus $25 \%$ ). The frequency of DR4,X, however, did not differ between the groups. DR phenotypes without the risk antigens DR 3 or DR4 were more frequent in ICA-negative relatives than in IDDM relatives ( $17 \%$ versus $3 \%, p<0.025)$; the frequency in ICA-positive relatives $(4 \%)$ was similar to that in IDDM relatives. There was no significant difference between the frequencies of DR phenotypes in IDDM relatives and a panel of 270 unrelated IDDM subjects (Table 2 ).

The frequency of DQ8 was increased with a corresponding decrease in the frequency of DQ7 in the three groups of relatives compared with unrelated controls (DQ8, $p<0.05-<0.005$; DQ7, $p<0.05<0.01$, Table 1 ). The frequency of DQ8 was lower in ICA-negative than in IDDM relatives $(38 \%$ versus $59 \%, p<0.025)$ with ICApositive relatives being intermediate $(45 \%)$ (i.e., not significantly different to either group), whereas the frequency of DQ7 did not differ between groups $(21 \%$ versus $20 \%$ versus $19 \%)$. The frequency of DQ2 did not differ between unrelated controls and ICA-negative relatives $(46 \%$ versus $51 \%$ ) or between ICA-positive and IDDM relatives $(68 \%$ versus $68 \%)$ but was higher than unrelated controls in ICA-positive and IDDM relatives $(p<$ $0.005,<0.005)$ and higher than ICA-negative relatives in IDDM relatives $(p<0.05)$. Only in the ICA-positive relatives was DQ2 higher than DQ8 ( $68 \%$ versus $45 \%, p<0.05)$. The frequency of DQ6 did not differ between unrelated controls and ICA-negative relatives ( $10 \%$ versus $11 \%$ ) but was lower than both in the IDDM relatives $(1 \%, p<$ $0.05)$, with ICA-positive relatives being intermediate $(6 \%)$.

\section{HLA class I alleles}

Most HLA class I antigens did not differ in frequency between relatives. The frequency of HLA-B8 was higher in each group of relatives than in unrelated controls $(p<0.025<0.005)$, reflecting the pattern of DR3 with which it is in linkage disequilibrium. However the frequencies of $\mathrm{B} 8$ in the ICA-negative, ICA-positive, and IDDM relatives ( $38 \%$ versus $47 \%$ versus $44 \%$ ) did not differ significantly.

The frequency of HLA-Al in unrelated controls did not differ from ICA-negative relatives ( $33 \%$ versus $39 \%$ ), but was lower than in ICApositive relatives $(55 \%, p<0.005)$ and IDDM relatives $(46 \%, p<0.05)$. The frequency of HLA-A2 did not differ between unrelated controls and ICA-negative or positive relatives $(50 \%$ versus $48 \%$ versus $53 \%$ ), but was lower in
IDDM relatives than in controls $(34 \%, p<0.05)$. However, these $p$ values did not remain significant following correction.

Unexpectedly, the frequency of HLA-A24 was higher in the IDDM relatives than in unrelated controls $(29 \%$ versus $16 \%, p<0.05)$ and even ICA-positive relatives $(9 \%, p<0.025)$ (Table 1) but was higher in ICA-negative relatives than in ICA-positive relatives $(24 \%$ versus $9 \%, p$ $<0.05$ ). During 3 years of follow-up, eight ICApositive relatives developed IDDM. Of these, five (63\%) had A24 compared with 5/53 (9\%, $p<$ 0.0005 ) of the remaining ICA-positive relatives who did not develop IDDM over the same period. HLA-A24 was not significantly associated with HLA-B alleles in specific haplotypes. The DR phenotype with which it occurred varied in each group of relatives (Table 3 ). DR4 occurred more frequently in A24 phenotypes in IDDM relatives $(16 / 20,80 \%)$ than in ICA-negative relatives $(10 /$ $23,43 \%, p<0.05)$, but not in ICA-positive relatives $(2 / 4,50 \%, p<0.1)$. In the ICA-negative relatives, A24 occurred predominantly with DRX,X $(7 / 16,44 \%), D R 4, X(7 / 30,23 \%)$, and DR3, $\mathrm{X}(6 / 27,22 \%)$, whereas in the IDDM relatives it occurred more frequently with DR3,4 $(10 / 30,33 \%), \mathrm{DR} 4, \mathrm{X}(5 / 14,39 \%)$ and DR3,X $(3 / 7,43 \%)$. Because the number of relatives in each phenotype group was too small to allow statistical comparison, the phenotypes of the panel of unrelated IDDM subjects (Table 2 ) were examined for the frequency of A24 (Table 4). The highest frequency of A24 (26\%) was in the DRX, $X$ subjects but again it was not significantly different than in other phenotype groups. However, when age of diabetes mellitus diagnosis in each phenotype group was analyzed, those with DR4,4 and DR3,X but not DR3,4 phenotypes had significantly younger ages of onset if they also had A24 (DR4,4 $p<0.005$; DR3,X $p<0.05$, Table 4).

\section{DISCUSSION}

To determine if specific HLA antigens identify individuals more likely to develop islet antibodies or progress to IDDM, we compared HLA antigen frequencies between ICA-negative, ICApositive, and IDDM first degree relatives. HLA antigen frequencies in relatives of patients with IDDM differ from those in unrelated controls, but in first-degree relatives should be biased towards similarity. Differences between relatives would, therefore, be of interest. Significant dif- 
TABLE 3. HLA-A24 frequency in DR phenotypes

\begin{tabular}{|c|c|c|c|c|c|c|}
\hline \multirow[b]{3}{*}{ Phenotype } & \multicolumn{6}{|c|}{ IDDM Family Group } \\
\hline & \multicolumn{2}{|c|}{$\begin{array}{c}\text { ICA-Negative } \\
(n=96)\end{array}$} & \multicolumn{2}{|c|}{$\begin{array}{c}\text { ICA-Positive } \\
\quad(n=53)\end{array}$} & \multicolumn{2}{|c|}{$\begin{array}{l}\text { IDDM } \\
(n=68)\end{array}$} \\
\hline & $n$ & $\%$ & $n$ & $\%$ & $n$ & $\%$ \\
\hline $\mathrm{DR} 3,4$ & $1 / 14$ & 7 & $0 / 16$ & 0 & $10 / 30$ & 33 \\
\hline DR 3,3 & $0 / 4$ & 0 & $0 / 3$ & 0 & $1 / 7$ & 15 \\
\hline DR4,4 & $2 / 5$ & 40 & $0 / 4$ & 0 & $1 / 8$ & 13 \\
\hline $\mathrm{DR} 4, \mathrm{X}$ & $7 / 30$ & 23 & $2 / 15$ & 13 & $5 / 14$ & 39 \\
\hline DR3,X & $6 / 27$ & 22 & $2 / 13$ & 15 & $3 / 7$ & 43 \\
\hline $\mathrm{DRX}, \mathrm{X}$ & $7 / 16$ & 44 & $0 / 2$ & 0 & $0 / 2$ & 0 \\
\hline
\end{tabular}

ferences were revealed in HLA antigen frequencies between relatives, depending on whether they were positive for ICA (i.e., had evidence of islet autoimmunity denoting an increased risk for IDDM) or had established IDDM.

As might be expected, the class II antigens DR3, DR4, DQ2, and DQ8 together identified relatives most likely to develop both ICA and IDDM. DR2, and to a lesser extent DQ6, identified those least likely. DR4 and DQ8 on one or both haplotypes identified ICA-positive relatives most likely to develop IDDM, whereas DR3 and DQ2 identified those likely to develop IDDM, only if present on both haplotypes. Considering DR phenotypes, relatives with DR3,4 were most likely to develop ICA and IDDM, relatives with DR3, X developed ICA but were least likely to develop IDDM, and relatives with DRX,X were the least likely to develop ICA and IDDM.

IDDM is considered to be a $\mathrm{T}$ cell-mediated disease in which antibodies have no primary pathogenic role (1). In a previous study in at-risk ICA-positive relatives, we found that DR3 was associated with high levels of antibodies but not proliferative CD4 T-cell responses to the islet autoantigen glutamic acid decarboxylase (GAD) (10). In the current study, the frequency of the DR3,X phenotype was 2.5 times lower in IDDM subjects than in their ICA-positive relatives $(10 \%$ versus $25 \%$ ). In contrast, the frequency of the DR4, X phenotype was unchanged between these groups ( $21 \%$ versus $28 \%$ ) and, after DR3,4, was the highest frequency DR phenotype in both IDDM relatives and unrelated IDDM subjects.

TABLE 4. HLA-A24 frequency in DR phenotypes and age at diagnosis of diabetes mellitus: unrelated IDDM subjects

\begin{tabular}{ccccc}
\hline & \multicolumn{2}{c}{$\begin{array}{c}\text { Frequency } \\
\text { HLA-A24 }\end{array}$} & & \multicolumn{1}{c}{$\begin{array}{c}\text { Mean Age } \\
\text { at Diagnosis of Diabetes }\end{array}$} \\
\cline { 2 - 5 } Phenotype & $n$ & $\%$ & A24+ & $15.9 \pm 10.3$ \\
\hline DR3,4 & $21 / 107$ & 20 & $13.1 \pm 5.8$ & $16.5 \pm 11.2$ \\
DR3,3 & $2 / 26$ & 8 & $24.5 \pm 9.5$ & $20.4 \pm 10.9^{a}$ \\
DR4,4 & $4 / 24$ & 17 & $7.0 \pm 2.9$ & $20.4 \pm 12.5$ \\
DR4,X & $11 / 64$ & 17 & $13.9 \pm 10.4$ & $24.3 \pm 11.5^{b}$ \\
DR3,X & $3 / 22$ & 14 & $11.7 \pm 6.8$ & $24.3 \pm 12.3$ \\
DRX,X & $7 / 27$ & 26 & $31.1 \pm 11.9$ & \\
\hline
\end{tabular}

$p$ values, Wilcoxon rank sum test, ${ }^{a}<0.005,{ }^{b}<0.05$. 
Thus, this within-family analysis confirms that DR4 confers a greater risk of IDDM than DR3.

The HLA class I antigen, HLA-A24, occurred more frequently in IDDM than in ICA-positive relatives. If A24 were an additional risk factor for IDDM, it might be expected that its frequency would be normal in ICA-negative relatives, intermediate in ICA-positive relatives, and high in IDDM subjects. If, however, the risk only acted at the later stages of the preclinical phase (i.e., after the development of ICA), a relatively faster loss of A24-positive, ICA-positive subjects into the IDDM group of relatives would be expected. At the time of data collection for this analysis, seven ICA-positive subjects had developed IDDM, four of whom had A24; subsequently, one further A24-positive ICA-positive subject developed IDDM. Thus, A24 was significantly more frequent in the ICA-positive relatives who developed IDDM than in those who did not. This implies that A24 is an additional risk factor for IDDM, but not for ICA positivity. The frequency of A24 was highest in the low frequency DR3, $X$ phenotype in IDDM relatives and in the low frequency DRX, X phenotype in the unrelated IDDM subjects (there were only two DRX,X phenotypes in the IDDM relatives). As the family data demonstrate, these DR phenotypes are themselves associated with very low risk for IDDM. The presence of A24 in subjects with these phenotypes who have developed IDDM indicates therefore that A24 confers additional independent risk. In this context, it may be relevant that of the five ICA-positive relatives with HLA-A24 who progressed to IDDM, only two had the high-risk class II HLA antigens DR3,4; DQ2,8. In addition, in the unrelated subjects, the age at diagnosis of diabetes mellitus was significantly lower in those with A24 and the phenotypes DR3,X; DR4,4. This effect of A24 was again seen only in association with class II phenotypes that confer less risk than DR3,4 or DQ2,8. This may be because the latter already predispose to disease onset at a younger age (Table 4) $(5,6)$.

A24 has been reported, together with A2 or $\mathrm{A} 3$ on the B62-DR4-DQ8 haplotype, to be more frequent in Finnish IDDM subjects than in controls (11). Insight into the significance of A24 comes from two studies of Japanese IDDM subjects $(12,13)$. In Japanese, IDDM is associated with DR4-DQ9, a submaximal susceptibility haplotype, and not with DR3-DQ2 as in Caucasoids, and is usually of adult onset. However, A24 in Japanese was significantly associated with a young age at diagnosis (10 versus 52 years), a shorter "honeymoon phase" of non-insulin de- pendency ( $<3$ months) and complete loss of residual insulin secretion within 1 year of diagnosis $(12,13)$. Our within-family analysis of relatives including those progressing to IDDM, as well as our cross-sectional analysis of unrelated IDDM subjects, demonstrates that A24 in Caucasoids also contributes to a more rapid and complete loss of $\beta$ cell function. We conclude that progressive $\beta$ cell destruction in some cases is class I restricted and mediated by CD8 $\mathrm{T}$ cells which target peptide(s) presented in A24 on $\beta$ cells. Further analysis of larger family groups might suggest a similar role for other class I antigens in association with particular class II phenotypes or haplotypes.

The present findings can be interpreted in the light of recent knowledge about binding of antigenic peptides to MHC molecules and the nature of the immune response evoked. Peptides that bind with high affinity may be more likely to drive differentiation of naive CD4 T cells towards TH1 cells which produce IFN- $\gamma$ and IL- 2 and provide help to CD8 $\mathrm{T}$ cells, whereas those that bind with low affinity may elicit TH2 cells which produce IL-4 and IL-5 and provide help to B cells to generate antibodies $(14,15)$. DR4 binds allelespecific peptides at nanomolar concentrations $(16,17)$. On the other hand, binding to DR3 requires a peptide motif distinct from that recognized by most other DR $\beta 1$ alleles (18), and DR3 binds peptides at micromolar concentrations $(18,19)$. Thus, autoantigenic peptides bound to DR4 could generate predominantly THl responses and those bound to DR3 predominantly TH2 responses. This hypothesis might explain the wellknown synergy between DR4 and DR3 in IDDM. DR4 would generate THI CD4 responses to a restricted set of high-affinity autoantigen peptides, followed by CD8 responses to autoantigen peptides presented by class I antigens. DR3 would generate TH2 responses to a wide range of low-affinity peptides encompassing those not only from the primary autoantigen(s) but also from proteins released from damaged $\beta$ cells. In the course of a TH2 response to multiple peptides, the generation of $B$ cells bearing specific antibodies would facilitate the efficient uptake and processing of proteins, peptides from which at high affinity and/or in sufficient density would be bound and presented by coexpressed DR4, to elicit TH1 responses. CD8 cells would then be able to target a wide range of epitopes in the context of a variety of class I alleles on beta cells. If HLA-A24 is particularly suited to binding peptides from a primary and/or dominant autoantigen, then A24-bound peptides on the beta 
cell could hasten the destructive process in individuals with either a less aggressive THl response (i.e., those with DR4,X, DR3,X, or DRX,X) or a TH1 response to a restricted set of peptides (i.e., those with DR4,4). However, in DR3,4 individuals in whom CD8 cells would recognize many peptides bound to whichever class I antigens were present, an effect of A24 would not be evident.

In conclusion, analysis of cohorts of normal, at-risk, and IDDM first-degree relatives reveals that HLA genes not only predispose to islet autoimmunity but also influence progression to clinical IDDM. These findings have implications for the prediction and prevention of IDDM and provoke a mechanistic hypothesis that could be tested experimentally.

\section{ACKNOWLEDGMENTS}

We thank Enzio Bonifacio for critical reading of the manuscript and we thank Katherine Kelly and Margaret Thompson for secretarial assistance. These studies were supported by the Victorian Health Promotion Foundation.

\section{REFERENCES}

1. Honeyman MC, Harrison LC. (1993) The immunological insult in type 1 diabetes mellitus. Springer Semin. Immunopathol. 14: 253-274.

2. Bingley PJ, Bonifacio E, Gale EAM. (1993) Can we really predict IDDMM? Diabetes 42: 213-220.

3. Ghosh S, Palmer SM, Rodrigues NR, et al. (1993) Polygenic control of autoimmune diabetes mellitus in nonobese diabetic mice. Nature Genet. 4: 404-409.

4. Tait BD, Harrison LC. (1991) Overview: The major histocompatibility complex and insulin dependent diabetes mellitus. Bailliere's Clin. Endocrinol. Metab. 5: 211-228.

5. Caillat-Zucman S, Garchon HJ, Timsit J, et al. (1992) Age-dependent genetic heterogeneity of type 1 insulin-dependent diabetes mellitus mellitus. J. Clin. Invest. 90: 2242-2250.

6. Tait BD, Harrison LC, Drummond BP, Stewart V, Varney $M$, Honeyman MC. (1995) HLA and age at diagnosis of insulindependent diabetes mellitus mellitus. Hum. Immunol. 42: 116-122.

7. Tarn AC, Thomas JA, Dean BM, et al. (1993) Predicting insulin-dependent diabetes mellitus. Lancet 1: 845-850.
8. Bingley PJ, Gale EAM. (1991) Lessons from family studies. Balliere's Clin. Endocrinol. Metab. 5: 211-228.

9. Kimura A, Sasazuki T. (1991) Protocol for HLA DNA typing technique. In: Tsuji $\mathrm{K}$, Aizawa M, Sasazuki T (eds). HLA 1991-Proceedings of the Eleventh International Histocompatibility Workshop and Conference. Oxford University Press, Oxford, pp. 397-419.

10. Harrison LC, Honeyman MC, De Aizpurua $\mathrm{HJ}$, et al. (1991) Inverse relationship between humoral and cellular immunity to glutamic acid decarboxylase in subjects at risk of insulin-dependent diabetes mellitus. Lancet 341: 1365-1369.

11. Fennessy $M$, Metcalfe $K$, Hitman $G$, et al. (1994) A gene in the HLA class I region contributes to susceptibility to IDDMM in the Finnish population. Diabetologia 37: 937-944.

12. Nakanishi K, Kobayashi T, Murasi T. (1993) Association of HLA-A24 with complete $\beta$-cell destruction in IDDMM. Diabetes 42: 1086-1093.

13. Kobayashi T, Tamemoto $K$, Nakanishi $K$, et al. (1993) Immunogenetic and clinical characterisation of slowly progressive IDDMM. Diab. Care 16: 780-788.

14. Pfeiffer C, Murray J, Madri J, Bottomly K. (1991) Selective activation of THI and TH2 like cells in vivo. Response to human collagen IV. Immunol. Rev. 123: 65-84.

15. Pfeiffer C, Stein J, Southwood S, Ketelaar H, Sette A, Bottomly K. (1995) Altered peptide ligands can control CD4 T lymphocyte differentiation in vivo. J. Exp. Med. 181: 1569-1574.

16. Hammer J, Belunis C, Bolin D, et al. (1994) High-affinity binding of short peptides to major histocompatibility class II molecules by anchor combinations. Proc. Natl. Acad. Sci. 91: 4456-4460.

17. Rothbard JB, Marshall K, Wilson KJ, Fugger L, Zaller D. (1994) Prediction of peptide affinity to HLA DRB1*0401. Int. Arch. Allergy Immunol. 105: 1-7.

18. Sidney J, Oseroff C, Southwood S, et al. (1992) $\mathrm{DRB} 1 * 0301$ molecules recognise a structural motif distinct from the one recognised by most DRB I alleles. J. Immunol. 149: 2634-2640.

19. Malcherek G, Gnau V, Stevanovic S, Rammensee HG, Jung G, Melms A. (1994) Analysis of allele-specific contact sites of natural HLA-DR 17 ligands. J. Immunol. 153: 1141-1149. 